\title{
Theoretical Analysis of Mass Transfer with Chemical Reaction Using Absorption of Carbon Dioxide into Phenyl Glycidyl Ether Solution
}

\author{
Subramaniam Muthukaruppan ${ }^{1}$, Indira Krishnaperumal ${ }^{2}$, Rajendran Lakshmanan ${ }^{2 *}$ \\ ${ }^{1}$ District Institute of Education and Training, Pudukkottai, India \\ ${ }^{2}$ Department of Mathematics, The Madura College, Madurai, India \\ Email: *raj_sms@rediffmail.com
}

Received August 16, 2012; revised September 16, 2012; accepted September 23, 2012

\begin{abstract}
Theoretical analysis corresponding to the diffusion and reaction kinetics in a chemical reaction between carbon dioxide and phenyl glycidyl ether solution is presented. Analytical expressions pertaining to the concentration of carbon dioxide $\left(\mathrm{CO}_{2}\right)$, phenyl glycidyl ether solution (PGE) and flux are obtained in terms of reaction rate constants. In this paper, a powerful analytical method, called the Adomian decomposition method (ADM) is used to obtain approximate analytical solutions for nonlinear differential equations. Furthermore, in this work the numerical simulation of the problem is also reported using Scilab/Matlab program. An agreement between analytical and numerical results is noted.
\end{abstract}

Keywords: Carbon Dioxide; Phenyl Glycidyl Ether Solution; Nonlinear Differential Equations; Adomian Decomposition Method; Boundary Value Problems

\section{Introduction}

Carbon dioxide is generally a useful gas that is made up of a carbon atom and two oxygen atoms. It is very important in plant photosynthesis, manufacturing carbonated soft drinks, powering pneumatic systems in robots, used in fire extinguisher, removing caffeine from coffee, etc., Carbon dioxide has the potential to provide a vast and cheap source of carbon. Turning it into useful products would also reduce its environmental impact as a greenhouse gas. Scientists have shown that ionic liquids are selective catalysts for converting carbon dioxide into synthetic intermediates called cyclic carbonates, but it is difficult to separate and recycle the liquid catalyst.

Recently, the chemical fixation of carbon dioxide has become an important research topic [1], because of the danger posed by global warming, and conversion of carbon dioxide into valuable substances is an extremely attractive solution. The reaction with oxiranes leading to five-membered cyclic carbonate (oxirane-reaction) is wellknown among many examples [2,3]. These carbonates can be used as aprotic polar solvent and sources for polymer synthesis [4]. In the oxirane-reaction, high pressure (5 - $50 \mathrm{~atm})$ of $\mathrm{CO}_{2}$ has been thought to be necessary [2]. The oxirane-reactions under atmospheric pressure have been reported [4].

Many organic and inorganic compounds including

"Corresponding author. ammines, phosphines, quaternary ammonium salts, and alkali metal salts are known to catalyze the oxiranereaction [3]. The kinetics of the reaction between $\mathrm{CO}_{2}$ and phenyl glycidyl ether (PGE) have been studied using catalyst THA-CP-MS41, The reaction rate constants were obtained using the measured absorption rate of and analyzed with the mass transfer mechanism associated with the chemical reactions.

Park et al. [5] investigated the chemical absorption of carbon dioxide and phenyl glycidyl ether solution containing the catalyst THA-CP-MS41 in a heterogeneous system. To our knowledge no analytical solutions of this model have been reported. The purpose of this communication is to derive simple approximate analytical expression for the steady-state concentrations of $\mathrm{CO}_{2}$, PGE and flux using the Adomian decomposition method.

\section{Mathematical Formulation of the Problem and Analysis}

Figure 1 shows the schematic representation of the stirred-cell absorber [5]. The overall reaction between $\mathrm{CO}_{2}$ and phenyl glycidyl ether (PGE) to form the 5-membered cyclic carbonate is as follows:

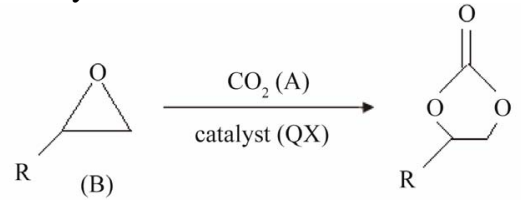




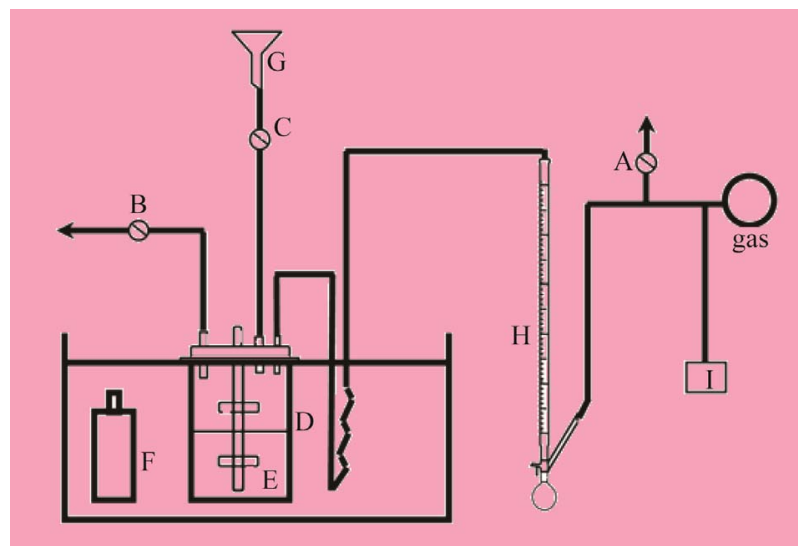

Figure 1. Shows the schematic representation of the stirredcell absorber [5]. A, B, C: Valve; D: Absorber; E: Impeller; F: Liquid bottle; G: Funnel; H: Soap film meter; I: Gas chromatography.

where $\mathrm{R}$ is a functional group of $-\mathrm{CH}_{2}-\mathrm{O}-\mathrm{C}_{6} \mathrm{H}_{5}$. The overall reaction of Equation (1) consists of two conescutive steps: 1) a reversible reaction between PGE (B) and THA-CP-MS41 (QX) to form an intermediate complex $\left.\left(C_{1}\right) ; 2\right)$ an reversible reaction between $C_{1}$ and $\mathrm{CO}_{2}$ (A) to form QX and five-membered cyclic carbonate (C):

$$
\begin{gathered}
\mathrm{B}+\mathrm{QX} \underset{k_{2}}{\stackrel{k_{1}}{\leftrightarrow}} \mathrm{C}_{1} \\
\mathrm{~A}+\mathrm{C}_{1} \stackrel{k_{3}}{\rightarrow} \mathrm{C}+\mathrm{QX}
\end{gathered}
$$

At steady state condition, the consecutive chemical reaction rate of $\mathrm{CO}_{2}$ to form $C_{1}$ is given as follows:

$$
r_{A, \text { cons }}=\frac{C_{B} S_{t}}{\frac{1}{k_{1}}+\frac{1}{K_{1} k_{3} C_{A}}+\frac{C_{B}}{k_{3} C_{A}}}
$$

where, $S_{t}$ is the surface area of catalyst, $C_{A}$ and $C_{B}$ are the concentration of $\mathrm{CO}_{2}$ and PGE respectively. $K_{1}$ is the reaction equilibrium constant, $k_{1}$ is the forward reaction rate constant in Equation (2) and $k_{3}$ is the forward reaction rate constant in Equation (3). The mass balances of $\mathrm{CO}_{2}$ and PGE, using film theory accompanied by the consecutive chemical reactions are given as follows [5]:

$$
\begin{aligned}
& D_{A} \frac{\mathrm{d}^{2} C_{A}}{\mathrm{~d} z^{2}}=\frac{C_{B} S_{t}}{\frac{1}{k_{1}}+\frac{1}{K_{1} k_{3} C_{A}}+\frac{C_{B}}{k_{3} C_{A}}} \\
& D_{B} \frac{\mathrm{d}^{2} C_{B}}{\mathrm{~d} z^{2}}=\frac{C_{B} S_{t}}{\frac{1}{k_{1}}+\frac{1}{K_{1} k_{3} C_{A}}+\frac{C_{B}}{k_{3} C_{A}}}
\end{aligned}
$$

where $D_{A}$ and $D_{B}$ are the diffusivity of $\mathrm{CO}_{2}$ and PGE respectively and $z$ is the distance. The boundary conditions are:

$$
\begin{array}{lll}
C_{A}=C_{A i}, \frac{\mathrm{d} C_{B}}{\mathrm{~d} z}=0 & \text { at } & z=0 \\
C_{A}=C_{A L}, C_{B}=C_{B o} \text { at } & z=z_{L}
\end{array}
$$

Equations (5), (6) and the boundary conditions (7) can be normalized by employing the following parameters:

$$
\begin{aligned}
& a=\frac{C_{A}}{C_{A i}} ; b=\frac{C_{B}}{C_{B o}} ; \\
& \alpha_{1}=\frac{z_{L}^{2} S_{t} C_{B o} K_{1} k_{3}}{D_{A}} ; \quad \alpha_{2}=\frac{z_{L}^{2} S_{t} C_{A i} K_{1} k_{3}}{D_{B}} ; \\
& \beta_{1}=\frac{C_{A i} K_{1} k_{3}}{k_{1}} ; \beta_{2}=\frac{C_{B o} K_{1} k_{1}}{k_{1}} ; x=\frac{z}{z_{L}}
\end{aligned}
$$

where, $a$, is the concentration of $\mathrm{CO}_{2}, b$ is the concentration of PGE, $\alpha_{1}, \alpha_{2}, \beta_{1}, \beta_{2}$ normalized parameters and $x$ is the dimensionless distance. Now the two nonlinear reaction/diffusion Equations (5), (6) in normalized form becomes as follows:

$$
\begin{aligned}
& \frac{\mathrm{d}^{2} a}{\mathrm{~d} x^{2}}=\frac{\alpha_{1} a b}{1+\beta_{1} a+\beta_{2} b} \\
& \frac{\mathrm{d}^{2} b}{\mathrm{~d} x^{2}}=\frac{\alpha_{2} a b}{1+\beta_{1} a+\beta_{2} b}
\end{aligned}
$$

The above Equations (8), (9) are the system of nonlinear differential equations. While no general method of solving these nonlinear problems has been proposed, several vigorous procedure such as Adomian decomposition method [6-10], Homotopy perturbation method [1115] and Homotopy analysis method [16-21] etc., have been analyzed. Here, Adomian decomposition method is used to solve these nonlinear differential equations. The boundary conditions becomes,

$$
\begin{aligned}
& a=1, \frac{\mathrm{d} b}{\mathrm{~d} x}=0 \text { at } x=0 \\
& a=k, b=1 \text { at } x=1
\end{aligned}
$$

where, $k=\frac{C_{A L}}{C_{A i}}$. The enhancement factor of $\mathrm{CO}_{2}$, defined as the ratio of the flux of $\mathrm{CO}_{2}$ with chemical reaction to that without chemical reaction is as follows,

$$
\beta=-\left(\frac{\mathrm{d} a}{\mathrm{~d} x}\right)_{x=0}
$$

\section{Analytical Solutions of Concentrations of $\mathrm{CO}_{2}$ and $\mathrm{PGE}$ under Steady-State Condition Using the Adomian Decomposition Method}

In this paper, the Adomian decomposition method (see Appendix A) is used to solve nonlinear differential equations. The ADM [6-10] yields, without linearization, per- 
turbation or transformation, an analytical solution in terms of a rapidly convergent infinite power series with easily computable terms. The basic principle of this method is described in Appendix A and detailed derivation of dimensionless concentration of $\mathrm{CO}_{2}$ and PGE, from the nonlinear Equations (8) and (9) are described in Appendix B. Using this method (refer Appendix B), we obtain the analytical expression corresponding to the concentrations of $\mathrm{CO}_{2}$ and PGE as follows:

$$
\begin{aligned}
& a(x)=(k-1) x+1+\frac{\alpha_{1} x}{2 \beta_{1}}(x-1)-\frac{\alpha_{1}\left(1+\beta_{2}\right)}{\beta_{1}^{3}(k-1)^{2}} \\
& {\left[\left(\log \left(1+\beta_{2}+\beta_{1}((k-1) x+1)-1\right)\right)\right.} \\
& \left(1+\beta_{2}+\beta_{1}((k-1) x+1)\right)-x\left(\log \left(1+\beta_{2}+\beta_{1} k\right)-1\right)(12) \\
& \left.\left(1+\beta_{2}+\beta_{1} k\right)\right]-\frac{\alpha_{1}\left(1+\beta_{2}\right)}{\beta_{1}^{3}(k-1)^{2}}\left[\log \left(1+\beta_{2}+\beta_{1}\right)-1\right] \\
& \left(1+\beta_{2}+\beta_{1}\right)(x-1) \\
& b(x)=1+\frac{\alpha_{2}}{2 \beta_{1}}\left(x^{2}-1\right)-\frac{\alpha_{2}\left(1+\beta_{2}\right)}{\beta_{1}^{3}(k-1)^{2}} \\
& {\left[\left(\log \left(1+\beta_{2}+\beta_{1}((k-1) x+1)-1\right)\right)\right.} \\
& \left(1+\beta_{2}+\beta_{1}((k-1) x+1)\right)-\left(\log \left(1+\beta_{2}+\beta_{1} k\right)-1\right) \\
& \left.\left(1+\beta_{2}+\beta_{1} k\right)\right]+\frac{\alpha_{2}\left(1+\beta_{2}\right)(x-1)}{\beta_{1}^{2}(k-1)} \log \left(1+\beta_{2}+\beta_{1}\right)
\end{aligned}
$$

From Equation (11), we obtain the flux as

$$
\begin{aligned}
\beta= & 1-k-\frac{\alpha_{1}\left(1+\beta_{2}\right)}{\beta_{1}^{3}(k-1)^{2}}\left[\left(\log \left(1+\beta_{2}+\beta_{1} k\right)-1\right)\right. \\
& \left.\left(1+\beta_{2}+\beta_{1} k\right)-\left(\log \left(1+\beta_{2}+\beta\right)_{1}-1\right)\left(1+\beta_{2}+\beta_{1}\right)\right] \\
+ & \frac{\alpha_{1}\left(1+\beta_{2}\right)}{\beta_{1}^{2}(k-1)} \log \left(1+\beta_{2}+\beta_{1}\right)+\frac{\alpha_{1}}{2 \beta_{1}}
\end{aligned}
$$

\section{Numerical Simulation}

The function pdex4 in Scilab/Matlab software which is a function of solving the initial-boundary value problems for the two reaction/diffusion equations is used to solve Equations (8) and (9). The normalized concentrations of $\mathrm{CO}_{2}$ and PGE are compared with simulation results in Figures 1 and 2. A satisfactory agreement is noted. The Scilab/Matlab program is also given in Appendix C. In Table 1, the simulation results compared with ADM results, the maximum error is $0.64 \%$.

\section{Results and Discussion}

Equations (12) and (13) represent the new closed and simple approximate analytical expressions of the normalized concentrations of $\mathrm{CO}_{2}$ and PGE for all values of parameters $\alpha_{1}, \alpha_{2}, \beta_{1}, \beta_{2}$ and $k$. The current response is given in Equation (14). The concentration profiles of $\mathrm{CO}_{2}$ and PGE are shown in Figures 2 and 3. The concentration of $\mathrm{CO}_{2}$ increases when the normalized parameter $k$ increases (refer Figure 2(a)). The concentration of $\mathrm{CO}_{2}$ decreases when the parameters $\beta_{1}$ and $\beta_{2}$ increases (refer Figures 2(b) and (c)). In Figure 3, the concentration of PGE decreases when the normalized parameter $\alpha_{2}$ or surface area of catalyst increases and diffusivity of PGE decreases. Equation (14) represents the normalized flux. The value of flux increases as the parameters $\beta_{1}$ and $\beta_{2}$ or reaction equilibrium constant increases (refer Figures 4(a) and (b)). In Figure 4(c), the value of

\begin{tabular}{|c|c|c|c|c|c|c|c|c|c|}
\hline \multirow[b]{2}{*}{$x$} & \multicolumn{3}{|c|}{$\beta_{1}=0.1$} & \multicolumn{3}{|c|}{$\beta_{1}=1$} & \multicolumn{3}{|c|}{$\beta_{1}=10$} \\
\hline & $\begin{array}{c}\text { This work } \\
\text { Equation (12) }\end{array}$ & $\begin{array}{l}\text { Numerical } \\
\text { simulation }\end{array}$ & Error \% & $\begin{array}{c}\text { This work } \\
\text { Equation (12) }\end{array}$ & $\begin{array}{l}\text { Numerical } \\
\text { simulation }\end{array}$ & Error \% & $\begin{array}{c}\text { This work } \\
\text { Equation (12) }\end{array}$ & $\begin{array}{l}\text { Numerical } \\
\text { simulation }\end{array}$ & Error \% \\
\hline 0.2 & 0.7722 & 0.7754 & 0.4144 & 0.7896 & 0.7906 & 0.1267 & 0.8132 & 0.8132 & 0.0000 \\
\hline 0.4 & 0.5746 & 0.5797 & 0.8876 & 0.5971 & 0.5988 & 0.2847 & 0.6299 & 0.6300 & 0.0159 \\
\hline 0.8 & 0.2451 & 0.2482 & 1.2648 & 0.2558 & 0.2569 & 0.4300 & 0.2736 & 0.2737 & 0.0366 \\
\hline \multirow[t]{2}{*}{1.0} & 0.1000 & 0.1000 & 0.0000 & 0.1000 & 0.1000 & 0.0000 & 0.1000 & 0.1000 & 0.0000 \\
\hline & Average & & 0.6356 & Average & & 0.2116 & Average & & 0.0125 \\
\hline
\end{tabular}
flux decreases as the parameters $\alpha_{1}$ or or surface area of catalyst increases and diffusivity of $\mathrm{CO}_{2}$ decreases.

\section{Conclusions}

This paper presents a theoretical treatment of carbon dioxide and phenyl glycidyl ether solution in chemical reaction. Also, we have discussed the mathematical models of $\mathrm{CO}_{2}$ absorption into the PGE solution. We have solved the nonlinear differential equations both analytically and numerically. The approximate analytical expressions for

Table 1. Comparison of normalized substrate concentration of $\mathrm{CO}_{2}(A(x))$ between Equation (12) and numerical simulation for various values of parameters $\beta_{1}$ when $\alpha_{1}=1, \beta_{2}=0.001, k=0.1$. 


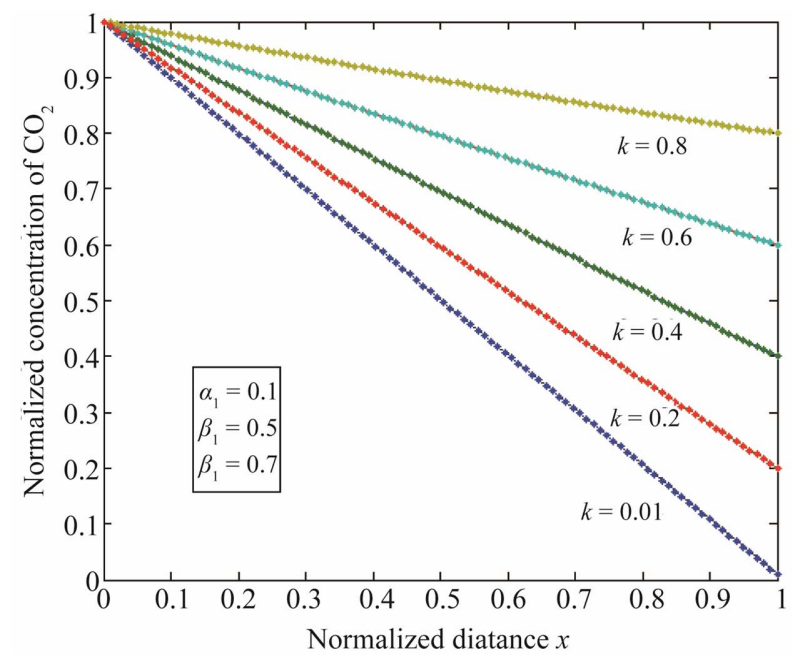

(a)

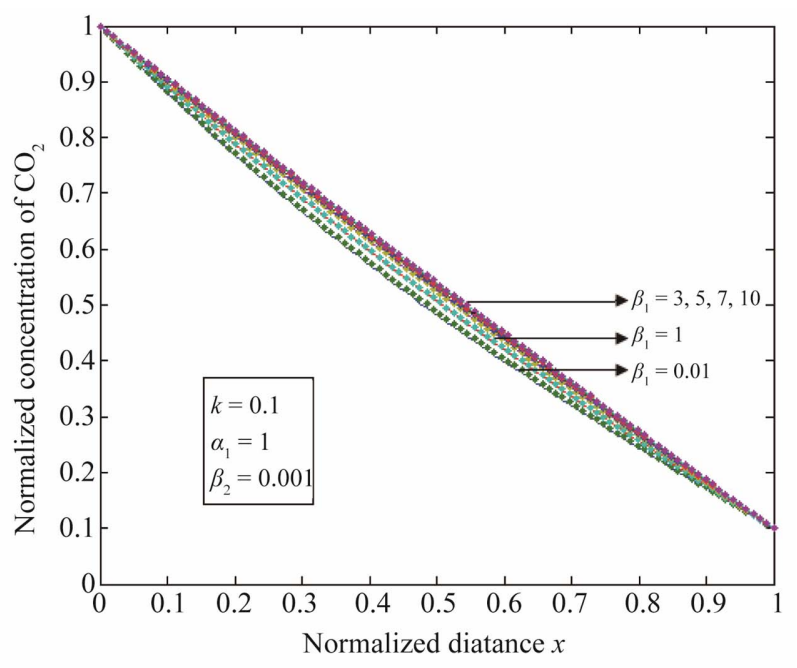

(b)

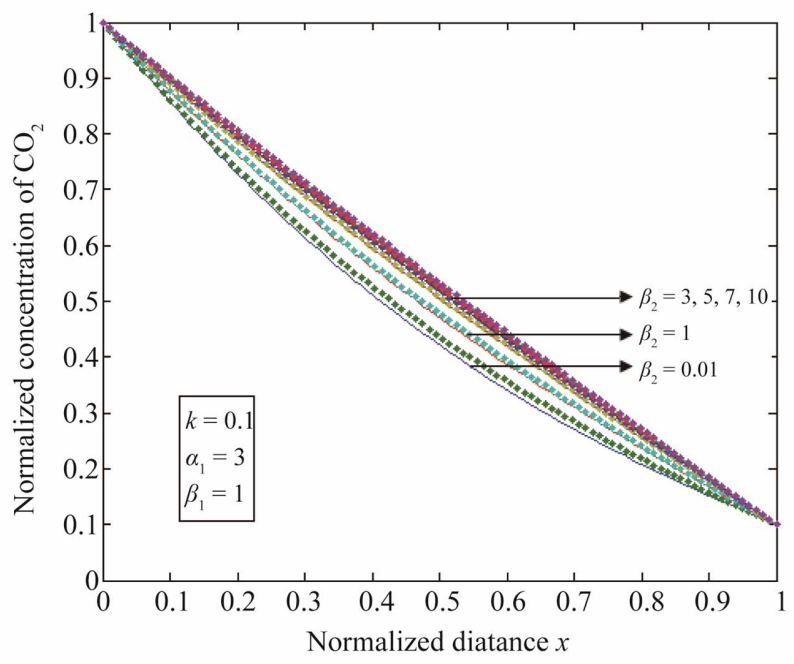

(c)

Figure 2. Normalized concentration of $\mathrm{CO}_{2}$ for various values of parameters is plotted using Equation (12). (a) $\alpha_{1}=0.1, \beta_{1}=$ $0.5, \beta_{2}=0.7$; (b) $k=0.1, \alpha_{1}=1, \beta_{2}=0.001$; (c) $k=0.1, \alpha_{1}=3, \beta_{1}=1$. The key to the graph (stacked line) represents the Equation (12) and (dotted line) represents the numerical simulation.

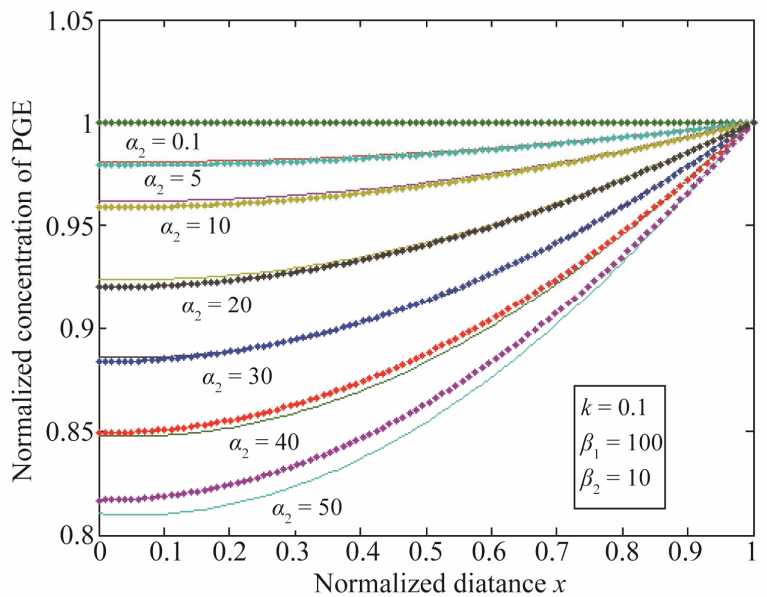

Figure 3. Normalized concentration of PGE for various values of parameters is plotted using Equation (13). The key to the graph (stacked line) represents the Equation (13) and (dotted line) represents the numerical simulation. 

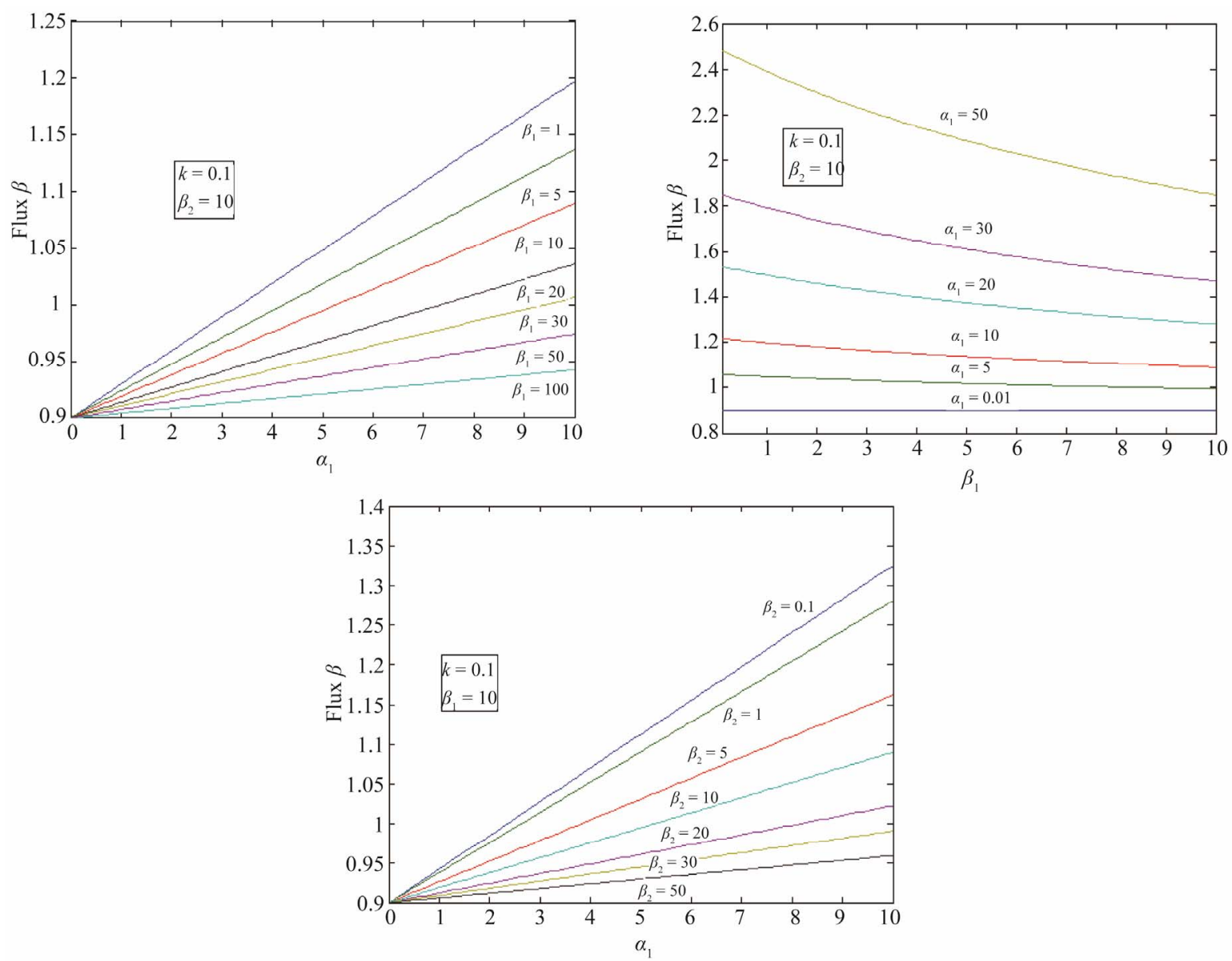

Figure 4. Diagrammatic representation of the normalized flux $\beta$ for various values of $\alpha_{1}, \beta_{1}, \beta_{2}$ and $k$.

the steady state concentrations of $\mathrm{CO}_{2}$ and PGE for all values of parameters using the Adomian decomposition method. These theoretical results are useful to evaluate the overall reaction rate constant and enhancement factor of $\mathrm{CO}_{2}$. A satisfactory agreement with the numerical result is noted.

\section{Acknowledgements}

This work was supported by the University Grants Commission (F. No. 39-58/2010(SR)), New Delhi, India. The authors are thankful to Dr. R. Murali, The Principal, The Madura College, Madurai and Mr. M. S. Meenakshisundaram, The Secretary, Madura College Board, Madurai for their encouragement. The author S. Muthukaruppan is very thankful to the Manonmaniam Sundaranar University, Tirunelveli for allowing to do the research work.

\section{REFERENCES}

[1] S. Inoue, "In Organic and Bioorganic Chemistry of Carbon Dioxide," In: S. Inoue and N. Amazaki, Eds., Kodansha Ltd., Tokyo, 1982.
[2] W. J. Peppel, "Preparation and Properties of the Alkylene Carbonates," Industrial and Engineering Chemical Research, Vol. 50, No. 5, 1958, pp. 767-770. doi:10.1021/ie50581a030

[3] N. Kihara, N. Hara and T. Endo, "Catalytic Activity of Various Salts in the Reaction of 2,3-Epoxypropyl Phenyl Ether and Carbon Dioxide under Atmospheric Pressure," The Journal of Organic Chemistry, Vol. 58, No. 23, 1993, pp. 6198-6202. doi:10.1021/jo00075a011

[4] G. Rokicki, "Cyclic Dicarbonates as New Monomers for the Synthesis of Poly(hydroxy ether)s," Die Makromolekulare Chemie, Vol. 186, No. 2, 1985, pp. 331-337. doi:10.1002/macp.1985.021860212

[5] Y. S. Choe, K. J. Oh, M. C. Kim and S. W. Park, “Chemical Absorption of Carbon Dioxide into Phenyl Glycidyl Ether Solution Containing THA-CP-MS41 Catalyst," Korean Journal of Chemical Engineering, Vol. 27, No. 6, 2010, pp. 1868-1875. doi:10.1007/s11814-010-0309-1

[6] G. Adomian, "Convergent Series Solution of Nonlinear Equations,” Journal of Computational and Applied Mathematics, Vol. 11, No. 2, 1984, pp. 225-230. doi:10.1016/0377-0427(84)90022-0

[7] A. Patela and S. E. Serrano, "Decomposition Solution of Multidimensional Groundwater Equations,” Journal of Hydrology, Vol. 397, No. 3-4, 2011, pp. 202-209. 


\section{doi:10.1016/j.jhydrol.2010.11.032}

[8] M. A. Mohamed, “Comparison Differential Transformation Technique with Adomian Decomposition Method for Dispersive Long-Wave Equations in (2+1)-Dimensions," Applications and Applied Mathematics, Vol. 5, No. 1, 2010, pp. 148-166.

[9] O. K. Jaradat, "Adomian Decomposition Method for Solving Abelian Differential Equations,” Journal of Applied Sciences, Vol. 8, No. 10, 2008, pp. 1962-1966. doi:10.3923/jas.2008.1962.1966

[10] A. M. Siddiquia, M. Hameed, B. M. Siddiquic and Q. K. Ghoric, "Use of Adomian Decomposition Method in the Study of Parallel Plate Flow of a Third Grade Fluid," Communications in Nonlinear Science and Numerical Simulation, Vol. 15, No. 9, 2010, pp. 2388-2399. doi:10.1016/j.cnsns.2009.05.073

[11] K. Indira and L. Rajendran, "Analytical Expression of Non Steady-State Concentration for the CE Mechanism at a Planar Electrode," Journal of Mathematical Chemistry, Vol. 50, No. 5, 2012 pp. 1277-1288. doi:10.1007/s10910-011-9968-3

[12] M. U. Maheswari and L. Rajendran, “Analytical Solution of Nonlinear Enzyme Reaction Equations Arising in Mathematical Chemistry," Journal of Mathematical Chemistry, Vol. 49, No. 8, 2011, pp. 1713-1726. doi:10.1007/s10910-011-9853-0

[13] A. Meena and L. Rajendran, "Mathematical Modeling of Amperometric and Potentiometric Biosensors and System of Nonlinear Equations-Homotopy Perturbation Approach," Journal of Electroanalytical Chemistry, Vol. 644, No. 1, 2010, pp. 50-59. doi:10.1016/j.jelechem.2010.03.027

[14] V. M. PonRani and L. Rajendran, "Mathematical Modelling of Steady-State Concentration in Immobilized Glucose Isomerase of Packed-Bed Reactors," Journal of Mathematical Chemistry, Vol. 50, No. 5, 2012, pp. 13331346. doi:10.1007/s10910-011-9973-6

[15] S. Anitha, A. Subbiah, S. Subramaniam and L. Rajendran, "Analytical Solution of Amperometric Enzymatic Reactions Based on Homotopy Perturbation Method,” Electrochimica Acta, Vol. 56, No. 9, 2011, pp. 3345-3352. doi:10.1016/j.electacta.2011.01.014

[16] S. J. Liao, "The Proposed Homotopy Analysis Technique for the Solution of Nonlinear Problems," Ph.D. Thesis, Shanghai Jiao Tong University, Shanghai, 1992.

[17] S. J. Liao, “On the Homotopy Anaylsis Method for
Nonlinear Problems,” Applied Mathematics and Computation, Vol. 147, No. 2, 2004, pp. 499-513. doi:10.1016/S0096-3003(02)00790-7

[18] S. J. Liao, "Comparison between the Homotopy Analysis Method and Homotopy Perturbation Method," Applied Mathematics and Computation, Vol. 169, No. 2, 2005, pp. 1186-1194. doi:10.1016/j.amc.2004.10.058

[19] S. J. Liao, "A New Branch of Solutions of BoundaryLayer Flows over an Impermeable Stretched Plate," International Journal of Heat and Mass Transfer, Vol. 48, No. 12, 2005, pp. 2529-2539. doi:10.1016/j.ijheatmasstransfer.2005.01.005

[20] S. J. Liao, "Beyond Perturbation: Introduction to the Homotopy Analysis Method,” CRC Press, Chapman \& Hall, Boca Raton, 2003. doi:10.1201/9780203491164

[21] S. J. Liao, "An Explicit, Totally Analytic Approximation of Blasius Viscous Flow Problems," International Journal of Non-Linear Mechanics, Vol. 34, No. 4, 1999, pp. 759-778. doi:10.1016/S0020-7462(98)00056-0

[22] A. M. Wazwaza and A. Gorguisb, “An Analytic Study of Fisher's Equation by Using Adomian Decomposition Method," Applied Mathematics and Computation, Vol. 154, No. 3, 2004, pp. 609-620. doi:10.1016/S0096-3003(03)00738-0

[23] J. Biazar and R. Islam, "Solution of Wave Equation by Adomian Decomposition Method and the Restrictions of the Method," Applied Mathematics and Computation, Vol. 149, No. 3, 2004, pp. 807-814. doi:10.1016/S0096-3003(03)00186-3

[24] N. H. Sweilama and M. M. Khaderb, “Approximate Solutions to the Nonlinear Vibrations of Multiwalled Carbon Nanotubes Using Adomian Decomposition Method," Applied Mathematics and Computation, Vol. 217, No. 2, 2010, pp. 495-505. doi:10.1016/j.amc.2010.05.082

[25] G. Adomian, "Solving the Mathematical Models of Neurosciences and Medicine," Mathematics and Computers in Simulation, Vol. 40, No. 1-2, 1995, pp. 107-114. doi:10.1016/0378-4754(95)00021-8

[26] G. Adomian, "Computation of Solutions to the Generalized Michaelis-Menton Equation,” Applied Mathematics Letters, Vol. 7, No. 4, 1994, pp. 45-48. doi:10.1016/0893-9659(94)90009-4

[27] O. D. Makinde, “Adomian Decomposition Approach to a SIR Epidemic Model with Constant Vaccination Strategy," Applied Mathematics and Computation, Vol. 184, No. 2, 2007, pp. 842-848. doi:10.1016/j.amc.2006.06.074 


\section{Appendix A}

\section{Basic Concept of the Adomian Decomposition Method (ADM)}

Adomian decomposition method [22-27] depends on decomposing the nonlinear differential equation

$$
F(\chi, y(\chi))=0
$$

into the two components

$$
L(y(\chi))+N(y(\chi))=0
$$

where $L$ and $N$ are the linear and the nonlinear parts of $F$ respectively. The operator $L$ is assumed to be an invertible operator. Solving for $L(y(\chi))$ leads to

$$
L(y(\chi))=-N(y(\chi))
$$

Applying the inverse operator $L$ on both sides of Equation (A.3) yields

$$
y(\chi)=\varphi(\chi)-L^{-1}[N(y(\chi))]
$$

where $\varphi(\chi)$ is a function that satisfies the condition $L(\varphi(\chi))=0$. Now assuming that the solution $y$ can be represented as infinite series of the form,

$$
\sum_{n=0}^{\infty} y_{n}(\chi)=\varphi(\chi)-L^{-1}\left(\sum_{n=0}^{\infty} A_{n}(\chi)\right)
$$

where

$$
\begin{aligned}
& \sum_{n=0}^{\infty} y_{n}(\chi)=y(\chi) \\
& A_{n}(\chi)=\frac{1}{n !}\left[\frac{\mathrm{d}^{n}}{\mathrm{~d} \lambda^{n}} N\left(\sum_{i=0}^{\infty}\left[\lambda^{i} y_{i}(\chi)\right]\right)\right]_{\lambda=0} \\
& \text { and } \sum_{n=0}^{\infty} A_{n}(x)=N(y(\chi)) \quad n \geq 0
\end{aligned}
$$

Then equating the terms in the linear system of Equation (A.5) gives the recurrent relation

$$
y_{0}=\varphi(\chi), \quad y_{n+1}=-L^{-1}\left(A_{n}\right) \quad n \geq 0
$$

However, in practice all the terms of series in Equation (A.5) cannot be determined, and the solution is approximated by the truncated series $\sum_{n=0}^{N} y_{n}(\chi)$.

\section{Appendix B}

\section{Analytical Expression of Concentrations of $\mathrm{CO}_{2}$ and PGE Using the Adomian Decomposition Method}

To solve Equations (8) and (9) using the Adomian decomposition method, we write the Equations (8) and (9) in the operator form,

$$
\begin{aligned}
& L[a(x)]=N[a(x)] \\
& L[b(x)]=N[b(x)]
\end{aligned}
$$

where

$$
\begin{aligned}
& L=\frac{\mathrm{d}^{2}}{\mathrm{~d} x^{2}}, N[a(x)]=\left[\frac{\alpha_{1} a b}{1+a \beta_{1}+b \beta_{2}}\right] \\
& N[b(x)]=\left[\frac{\alpha_{2} a b}{1+a \beta_{1}+b \beta_{2}}\right]
\end{aligned}
$$

Applying the inverse operator $L^{-1}$ on both sides of Equation (B.1) and (B.2) yields

$$
\begin{aligned}
& a(x)=A x+B+L^{-1} N[a(x)] \\
& b(x)=A x+B+L^{-1} N[b(x)]
\end{aligned}
$$

According to the ADM, the solution $a(x)$ and $b(x)$ can be elegantly computed by using the recurrence relation

$$
\begin{aligned}
& a_{0}(x)=A x+B, \\
& a_{\mathrm{n}+1}(x)=L^{-1} N[a(x)]=L^{-1} A_{n}(x), \\
& n \geq 0 \\
& b_{0}(x)=A x+B, \\
& b_{\mathrm{n}+1}(x)=L^{-1} N[b(x)]=L^{-1} B_{n}(x), \\
& n \geq 0
\end{aligned}
$$

where $A_{n}$ and $B_{n}$ are the Adomian polynomials of $a_{1}, a_{2} \cdots a_{n}$ and $b_{1}, b_{2} \cdots b_{n}$ respectively. We can find the first few Adomian polynomial coefficients $A_{n}$ and $B_{n}$ using Equation (A.6) as follows:

$$
\begin{aligned}
& A_{0}(x)=N\left(a_{0}\right)=\left[\frac{\alpha_{1} a_{0} b_{0}}{1+\beta_{1} a_{0}+\beta_{2} b_{0}}\right] \\
& B_{0}(x)=N\left(b_{0}\right)=\left[\frac{\alpha_{2} a_{0} b_{0}}{1+\beta_{1} a_{0}+\beta_{2} b_{0}}\right]
\end{aligned}
$$

The remaining polynomials $A_{\mathrm{i}}(x)$ and $B_{\mathrm{i}}(x)$ can be generated easily, using Equation (A.6). Applying the following boundary conditions

$$
\begin{aligned}
& a_{0}(0)=1, a_{0}(1)=k \\
& \text { and } a_{i}(0)=0, a_{i}(1)=0 \\
& \text { for } i \geq 1 \\
& b_{0}^{\prime}(0)=0, b_{0}(1)=1 \\
& \text { and } b_{i}^{\prime}(0)=0, b_{i}(1)=0 \\
& \text { for } i \geq 1
\end{aligned}
$$

From Equations (B.6) and (B.7) using the above conditions we obtain the following results:

$$
a_{0}(x)=(k-1) x+1
$$




$$
\begin{aligned}
& a_{1}(x) \\
& =\frac{\alpha_{1} x}{2 \beta_{1}}(x-1)-\frac{\alpha_{1}\left(1+\beta_{2}\right)}{\beta_{1}^{3}(k-1)^{2}} \\
& {\left[\left(\log \left(1+\beta_{2}+\beta_{1}((k-1) x+1)-1\right)\right)\right.} \\
& \left(1+\beta_{2}+\beta_{1}((k-1) x+1)\right)-x\left(\log \left(1+\beta_{2}+\beta_{1} k\right)-1\right) \\
& \left.\left(1+\beta_{2}+\beta_{1} k\right)\right]-\frac{\alpha_{1}\left(1+\beta_{2}\right)}{\beta_{1}^{3}(k-1)^{2}}\left[\log \left(1+\beta_{2}+\beta_{1}\right)-1\right] \\
& \left(1+\beta_{2}+\beta\right)_{1}(x-1) \\
& b_{0}(x)=1 \\
& b_{1}(x)=\frac{\alpha_{2}}{2 \beta_{1}}\left(x^{2}-1\right)-\frac{\alpha_{2}\left(1+\beta_{2}\right)}{\beta_{1}^{3}(k-1)^{2}} \\
& {\left[\left(\log \left(1+\beta_{2}+\beta_{1}((k-1) x+1)-1\right)\right)\right.} \\
& \left(1+\beta_{2}+\beta_{1}((k-1) x+1)\right)-\left(\log \left(1+\beta_{2}+\beta_{1} k\right)-1\right) \\
& \left.\left(1+\beta_{2}+\beta_{1} k\right)\right]+\frac{\alpha_{2}\left(1+\beta_{2}\right)(x-1)}{\beta_{1}^{2}(k-1)} \log \left(1+\beta_{2}+\beta_{1}\right)
\end{aligned}
$$

Adding Equations (B.12) and (B.13), (B.14) and (B.15), we get the concentration of $\mathrm{CO}_{2}$ and PGE (Equations (12) and (13)) in the text.

\section{Appendix C}

Scilab/Matlap program for the numerical solution of the system of nonlinear Equations (8) and (9)

function pdex 4

$\mathrm{m}=0$;

$\mathrm{x}=$ linspace $(0,1)$;

$\mathrm{t}=$ linspace $(0,100000)$;

sol=pdepe(m,@pdex4pde,@pdex4ic,@pdex4bc,x,t);

$\mathrm{u} 1=\operatorname{sol}(:,:, 1)$;

$\mathrm{u} 2=\operatorname{sol}(:,:, 2)$;

figure

plot(x,u1(end,:))

title('u1(x,t)')

xlabel('Distance $\mathrm{x}$ ')

ylabel('u1(x,2)')

figure

$\operatorname{plot}(\mathrm{x}, \mathrm{u} 2(\mathrm{end}, \mathrm{:}))$

title('u2(x,t)')

xlabel('Distance $\mathrm{x}$ ')

ylabel('u2(x,2)') function $[\mathrm{c}, \mathrm{f}, \mathrm{s}]=\operatorname{pdex} 4 \mathrm{pde}(\mathrm{x}, \mathrm{t}, \mathrm{u}, \mathrm{DuDx})$

$\mathrm{C}=[1 ; 1]$;

$\mathrm{f}=[1 ; 1] *^{*} \mathrm{DuDx}$;

$\mathrm{a}=0.001$;

$\mathrm{b}=10$;

$\mathrm{M}=10$;

$\mathrm{N}=0.01$;

$\mathrm{F}=-(\mathrm{a} * \mathrm{u}(1) * \mathrm{u}(2)) /(1+(\mathrm{M} * \mathrm{u}(1))+(\mathrm{N} * \mathrm{u}(2)))$;

$\mathrm{F} 1=-(\mathrm{b} * \mathrm{u}(1) * \mathrm{u}(2)) /(1+(\mathrm{M} * \mathrm{u}(1))+(\mathrm{N} * \mathrm{u}(2)))$;

$\mathrm{s}=[\mathrm{F} ; \mathrm{F} 1]$;

function $\mathrm{u} 0=\operatorname{pdex} 4 \mathrm{ic}(\mathrm{x})$;

$\mathrm{u} 0=[1 ; 1]$;

function [pl,ql,pr,qr]=pdex4bc(xl,ul,xr,ur,t)

$\mathrm{pl}=[\mathrm{ul}(1)-1 ; 0]$;

$\mathrm{ql}=[0 ; 1]$;

$\mathrm{pr}=[\operatorname{ur}(1)-0.1 ; \operatorname{ur}(2)-1]$;

$\mathrm{qr}=[0 ; 0]$;

\section{Appendix D}

\section{Nomenclature}

\section{Symbols}

$C_{A}$ : Concentration of $\mathrm{CO}_{2}(\mathrm{M})$

$C_{B}$ : Concentration of PGE (M)

$D_{A}$ : Diffusivity of $\mathrm{CO}_{2}\left(\mathrm{~m}^{2} / \mathrm{s}\right)$

$D_{B}$ : Diffusivity of PGE $\left(\mathrm{m}^{2} / \mathrm{s}\right)$

$K_{1}$ : Reaction equilibrium constant $(1 / \mathrm{M})$

$k_{1}$ : Forward reaction rate constant in reaction Equation (2) $\left(1 / \mathrm{m}^{2} \mathrm{~s}\right)$

$k_{2}$ : Backward reaction rate constant in reaction Equation

(2) $\left(\mathrm{M} / \mathrm{m}^{2} \mathrm{~s}\right)$

$k_{3}$ : Forward reaction rate constant in reaction Equation (3) $\left(1 / \mathrm{m}^{2} \mathrm{~s}\right)$

$r_{A, \text { cons }}$ : Reaction rate of $\mathrm{CO}_{2}$ in consecutive reaction model (M/s)

$S_{t}$ : Surface area of catalyst $\left(\mathrm{m}^{2}\right)$

$z$ : Distance (m)

$z_{L}$ : Film thickness $(\mathrm{m})$

$a$ : Normalized concentration of $\mathrm{CO}_{2}$ (Dimensionless)

$b$ : Normalized concentration of PGE (Dimensionless )

$\alpha_{1}, \alpha_{2}, \beta_{1}, \beta_{2}$ and $k$ : Normalized parameters (Dimensionless)

$\beta$ : Flux of $\mathrm{CO}_{2}$ (Dimensionless)

\section{Subscripts}
A: $\mathrm{CO}_{2}$
B: PGE
L: Bulk solution
o: Feed or solvent 